\title{
IACUC approval of SOPs
}

There is a burning desire within the hearts of some animal facility managers to have the IACUC approve each of the facility's Standard Operating Procedures (SOPs), every informal operating policy, every food ration used, and so forth. This is balanced by an equally strong desire of other facility managers to work cordially but at an arm's length from the IACUC. In the latter managers' opinions, the job of the IACUC is to oversee the program of animal care and use as it relates to federal regulations, and nothing more. Not surprisingly, both groups have trouble seeing the other's viewpoint.

At Great Eastern University, the difference in opinion was not among animal facility managers but between the facility director and the IACUC chairman. Dr. Bernard Koul, the animal facility director, believed that the IACUC had an obligation to assure that husbandry and veterinary care for laboratory animals met or exceeded the minimum standards of the Guide for the Care and Use of Laboratory Animals (the Guide) ${ }^{1}$ and the Animal Welfare Act regulations ${ }^{2}$. He had no problem with the IACUC reading the animal facility's SOPs and offering suggestions for improvements, but he did not believe that the IACUC had any authority to approve those SOPs or require changes that went beyond federal regulations. He quoted the section of the Animal Welfare Act regulations that states that an IACUC is to evaluate the care, treatment, housing and use of animals and to certify compliance with the Animal Welfare $\mathrm{Act}^{3}$. Koul also said that section 495 of the Health Research Extension Act of 1985 (which requires an IACUC for research funded by the Public Health Service $)^{4}$ said nearly the same thing and was focused on compliance with the Act, not on the approval of SOPs by the IACUC.

Dr. Larry Covelli, the IACUC chairman, disagreed with Koul. Covelli said the Health Research Extension Act and the Guide required the IACUC to oversee and evaluate animal care and use activities. The Animal
Welfare Act regulations, he said, required a similar review and also referred to animal activities, not just research protocols. In his opinion, approving SOPs that focused on animal activities were well within the scope of the IACUC's responsibility and authority.

Both Koul and Covelli concurred that the IACUC had an oversight and evaluation responsibility for husbandry and veterinary care issues but, using the same documents for their justification, they disagreed on the issue of the IACUC's authority to approve SOPs. What is your opinion and how would you resolve this impasse?

1. Committee for the Update of the Guide for the Care and Use of Laboratory Animals. Guide for the Care and Use of Laboratory Animals. 8th edn. (National Research Council, Washington, DC, 2011).

2. Animal Welfare Act. Public Law 89-544. 7 U.S.C. 2131 et seq.

3. Animal Welfare Act Regulation. 9 CFR, Part 1. Definitions: Committee.

4. Health Research Extension Act of 1985. Public Law 99-158. Sec. 495.

\section{RESPONSE}

\section{Review animal welfare SOPs}

\section{Cindy Horner, BS, CPIA, CMAR, RLATG \& Marie McKeon, MPhil, DABT}

Should IACUCs as mandated by the Animal Welfare Act (AWA) ${ }^{1}$, the Public Health Service Policy on Humans Care and Use of Laboratory Animals (PHS Policy) ${ }^{2}$ and the eighth edition of the Guide for the Care and Use of Laboratory Animals (the Guide $)^{3}$ have the authority to approve Standard Operating Procedures (SOPs)? Our IACUC is currently reviewing this question. The IACUC members, fully familiar with federal regulations and the Guide, are an institution's subject matter experts. The IACUC provides regulatory compliance guidance; serves as a resource to facility management on animal welfare issues; provides valuable input to the facility management concerning the interpretation of issues covered in the Guide; and provides scientific and ethical knowledge of a range of procedures.

The IACUC has a federal mandate in its function as described in the AWA and PHS Policy. Section 2.31 (4)(c)(1) of the AWA states that the IACUC should "review, at least once every six months, the research facility's program for humane care and use of animals..." 1 . Section 495(b)(1) of the PHS Policy requires "animal care committees at each entity which conducts biomedical and behavioral research with funds provided under this Act (including the US NIH and the national research institutes) to assure compliance with the guidelines established..."2 . The PHS Policy goes on to state in section 495(b)(3)(A) that the IACUC should "review the care and treatment of animals in all animal study areas and facilities of the research entity at least semiannually to evaluate compliance with applicable guidelines established..."2. In addition, the Guide states that "the IACUC is responsible for providing oversight and for evaluating the effectiveness of the training program"3.

The review and assurance of compliance of the animal program by the IACUC includes reviewing SOPs during the SemiAnnual Program and Facility Inspection at a minimum, or more often as necessary, as well as reviewing the policies by which an organization affirms its compliance with the AWA and the Guide and trains its staff. The IACUC, or at least one designated member (such as the veterinarian), 